\title{
Servicios ecosistémicos de masas mixtas de pino resinero y pino albar: conservación de la biodiversidad y secuestro de carbono
}

Ecosystem services of mixed stands of Scots pine and Maritime pine: biodiversity conservation and carbon sequestration

\author{
López-Marcos, D..$^{1,2,3, *}$
}

${ }^{1}$ Área de Ecología - Departamento de Ciencias Agroforestales. ETSIIAA de Palencia. Universidad de Valladolid.

${ }^{2}$ Área de Edafología y Química Agrícola - Departamento de Ciencias Agroforestales. ETSIIAA de Palencia. Universidad de Valladolid.

${ }^{3}$ Instituto Universitario de Investigación en Gestión Forestal Sostenible UVa - INIA. 


\section{Resumen}

La mayoría de estudios sobre los efectos positivos de la mezcla de árboles en el contexto de suministro de servicios ecosistémicos (SE) se centran en mezclas que combinan especies diferentes. Sin embargo, este efecto es poco conocido cuando se mezclan especies del mismo género. Por ello, evaluamos el efecto de la mezcla sobre el suministro de SE en seis tripletes [6 tripletes_3 rodales ( 2 rodales puros +1 rodal mixto $)=18$ parcelas) de pino albar (Pinus sylvestris L.) y pino resinero (P. pinaster Ait.) en el noroeste de España. En cada parcela, el suelo (una calicata de $50 \mathrm{~cm}$ de profundidad), el sotobosque (diez inventarios de $1 \times 1 \mathrm{~m}$ ) y el estrato arbóreo (a dos escalas espaciales; escala de vecindario: diez inventarios de $4 \mathrm{~m}$ de radio/parcela; escala de rodal: un inventario de $15 \mathrm{~m}$ de radio/parcela) fueron analizados.

Los resultados de esta tesis contribuyen a comprender los mecanismos involucrados en el suministro de SE en rodales puros vs. mixtos de $P$. sylvestris y $P$. pinaster: las masas mixtas presentan una ventaja competitiva sobre los rodales puros en términos de conservación de la biodiversidad, secuestro de carbono, fertilidad edáfica y producción del estrato arbóreo. Teniendo en cuenta que estas mezclas están ampliamente distribuidas en España, parece oportuno proponer que se sigan promoviendo ya contribuyen a incrementar la fertilidad del suelo, la acumulación de carbono, a proporcionar un exceso de área basal en relación con masas puras, y a conservar la regeneración de especies endémicas y mantenimiento de la riqueza del sotobosque en suelos con menor contenido hídrico.

Palabras clave: Pinus sylvestris, Pinus pinaster, servicios ecosistémicos

\section{Summary}

Most reports of the positive effects of tree mixture on the ecosystem services (ES) supply context focus on mixtures that combine tree species with contrasting traits. However, this effect is little known in mixed stands of species of the same genus. Thus, we evaluated the effect of mixing on ES supply in six triplets [6 triplets _ 3 forest stands ( 2 monospecific stands +1 mixed stand $)=18$ plots) of Scots pine (Pinus sylvestris L.) and Maritime pine (P. pinaster Ait.) in north-central Spain. In each plot, the soil (one pit soil of $50 \mathrm{~cm}$ depth), the understory (ten $1 \mathrm{x} 1 \mathrm{~m}$ random-inventories), and the overstory (at two spatial scales; neighborhood-scale: ten $4 \mathrm{~m}$-radius inventories/plot; stand-scale: one $15 \mathrm{~m}$-radius inventory/plot) were analyzed.

The results of this thesis contribute to understanding the mechanisms involved in the ES supply of the pure vs. mixed stands of $P$. sylvestris and $P$. pinaster: the mixtures present a competitive advantage over pure stands in terms of biodiversity conservation, carbon sequestration, soil fertility, and tree overstory production. Taking into account that these mixtures are widely distributed in Spain, it seems appropriate to propose that they continue to be promoted in the study area because they contribute to increasing the soil fertility, the subsoil carbon accumulation, providing a total basal area overyielding, conserving the regeneration of endemic species and keeping the richness of the understory in soils with lower water content.

Keywords: mixed stands, pure stands, Pinus sylvestris, Pinus pinaster, ecosystem services 


\section{Introducción}

Muchos estudios destacan el papel de los bosques mixtos vs. puros en el suministro de servicios ecosistémicos. La mayoría se centran en los efectos positivos de la mezcla de especies forestales con rasgos contrastables (i.e. frondosas-coníferas; Bravo-Oviedo et al., 2014; Del Río et al., 2015; Pretzsch et al., 2016). Pero poco se sabe sobre el efecto de la mezcla de especies del mismo género (pero ver Forrester et al., 2004, 2005, 2006a, b, Riofrío et al., 2017a, b, 2019; Cattaneo et al., 2018, 2020). Esta tesis doctoral evalúa el efecto de la mezcla en masas mixtas y puras de pino albar (Pinus sylvestris L.) y pino resinero (Pinus pinaster Aiton.) en la Sierra de la Demanda sobre el suministro de servicios ecosistémicos: Pero, ¿Qué son los servicios ecosistémicos?, ¿Qué son las masas mixtas? Y ¿Por qué estudiar las mezclas de pino resinero y pino albar en la Sierra de la Demanda?

\section{1. ¿Qué son los servicios ecosistémicos?}

Naciones Unidas en su informe sobre el estado de los ecosistemas terrestres, define a los servicios ecosistémicos como toda característica, proceso o función que de manera directa o indirecta contribuyen al bien estar humano (MEA, 2005). Aunque este término aparece antes en la bibliografía (Ehrlich and Mooney, 1983), su sentir estaba presente desde la segunda mitad del siglo XX dado que la pérdida de los mismos se hizo mucho más evidente (Beddoe et al., 2009).

Naciones Unidas (MEA, 2005), de manera muy general, divide todos estos servicios de ecosistémicos en cuatro categorías muy amplias:

— Servicios de provisión (i.e. madera).

- Servicios de regulación (i.e. regulación del clima).

— Servicios de soporte (i.e. ciclo de nutrientes).

- Servicios culturales (i.e. recreación del paisaje).

$\mathrm{Y}$ define diferentes sistemas o ecosistemas donde evaluarlos (i.e. sistemas costeros, de aguas continentales, polares, urbanos, bosques, islas, montañas, etc.). De todos estos ecosistemas Naciones Unidas destaca a los bosques dada la amplia gama de servicios ecosistémicos que proveen, como son la regulación del clima, la producción de madera, el secuestro del carbono, la conservación de la biodiversidad o la fertilidad edáfica.

\section{2. ¿Qué son las masas mixtas?}

Dada la amplia variabilidad de masas mixtas que encontramos en el planeta, se pueden encontrar diferentes definiciones en función de donde nos encontramos. En Europa, podemos seguir la definición derivada del proyecto "Eumixfor" que dice 
que una masa mixta es una unidad forestal, de al menos 0,5 ha de superficie, donde al menos dos especies forestales (en cualquiera de sus estados de desarrollo) coexisten y comparten recursos (Bravo-Oviedo et al., 2014).

\section{3. ¿Por qué estudiar las masas mixtas?}

El estudio y gestión de las masas mixtas ha cobrado mayor relevancia en las últimas décadas como resultado de la creciente evidencia de que las mezclas pueden suministrar servicios ecosistémicos de manera más eficiente que los bosques puros (Gamfeldt et al., 2013). Además, se les atribuye una amplia gama de beneficios como mayores tasas de crecimiento (Piotto, 2008), mejora en las condiciones edáficas (Davidson et al., 1998; Brandtberg et al., 2000) o el incremento de secuestro de carbono (Andivia et al., 2016; López-Marcos et al., 2018).

\section{4. ¿Existen masas mixtas en España?}

El antiguo MAGRAMA (2012) nos informa que entorno al 19\% de la superficie forestal española son masas mixtas. Masas mixtas compuestas por la mezcla de frondosas, la mezcla de fondosas-coniferas o la mezcla de coníferas (Riofrío, 2018).

\section{5. ¿Por qué estudiar las mezclas de pino resinero y pino albar en la Sierra de la Demanda?}

Esta tesis se centra en las mezclas de coníferas y en concreto en los pinares mixtos ya que suponen en torno a 500.000 ha de la superficie forestal española (Montero y Serrada, 2013). Estos pinares mixtos pueden estar compuestos por mezclas de diferentes especies: P. sylvestris-P. nigra J.F. Arnold (Trasobares et al., 2004), P. halepensis Mill.-P. nigra-P. sylvestris (Granda et al., 2018), P. halepensis-P. pinea L. (Cattaneo et al., 2018), P. pinaster-P. pinea (Ledo et al., 2014), P. sylvestris-P. nigra (Jucker et al., 2014), o P. pinaster-P. sylvestris (Riofrío et al., 2017a, b, 2019; Cattaneo et al., 2020; López-Marcos et al., 2018, 2019; 2020; 2021a, b).

Este último tipo, las mezclas de Pinus pinaster-Pinus sylvestris son el objeto de esta tesis doctoral ya que dos de las principales especies forestales de España (Fig. 1; Serrada et al., 2008). Estas especies crecen en rodales puros y mixtos ya sea de forma natural o como resultado de la selección de especies para la forestación (Serrada et al., 2008). Ambas especies conviven en pendientes moderadas principalmente en la Cordillera Ibérica y Central en aproximadamente 120.000 ha cuando se superponen sus distribuciones ecológicas naturales: en las zonas más frías y altas de distribución del pino resinero y cercanas al límite latitudinal sur de distribución euroasiática del pino albar (Riofrío, 2018).

Este estudio se realiza en la "Sierra de la Demanda", puesto que, además encontrar estas especies en masas tanto puras como mixtas, lo que nos permite hacer comparaciones por pares, es una zona donde hay una fuerte vinculación de la población local hacia el sector forestal. 

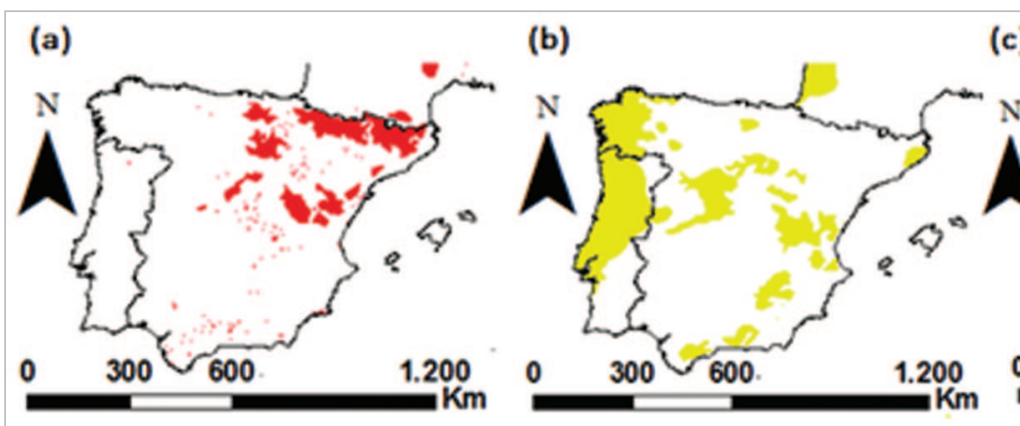

(c)

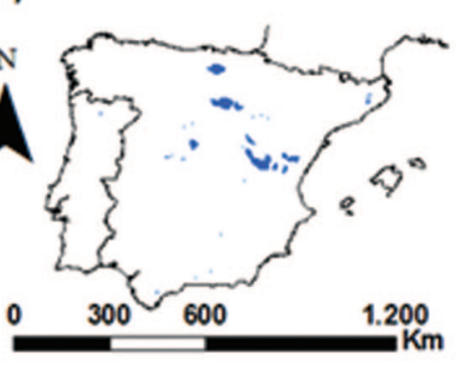

Figura 1. (a) Mapa de distribución del pino albar (Pinus sylvestris L.) (b) Mapa de distribución del pino resinero(Pinus pinaster Ait.) y (c) Mapa de distribución de mezclas de pino albar y pino resinero elaborado cruzando los datos del EUFORGEN (2009). (modificado de López-Marcos 2020).

\section{Objetivos}

El objetivo general de esta tesis doctoral fue aumentar el conocimiento sobre la dinámica y el funcionamiento de las masas puras $v s$. mixtas de pino resinero y pino albar en el contexto del suministro de servicios ecositémicos. Puesto que partíamos de la hipótesis de que estas masas mixtas podían ser más eficientes suministrando servicios ecosistémicos.

Para alcanzar este objetivo general en cada uno de los artículos originales que dio lugar a esta tesis doctoral se describieron diferentes objetivos parciales:

1. Cuantificar las diferencias entre los tipos de masas forestales el almacenamiento de carbono a lo largo del perfil edáfico (cada $10 \mathrm{~cm}$ de profundidad) y su relación con los cationes intercambiables, e investigar las posibles causas de estas diferencias observadas (López-Marcos et al., 2018).

2. Evaluar el efecto del estrato arbóreo sobre la riqueza del sotobosque y la composición de formas de vida de Raunkiær y su relación con las propiedades del suelo (López-Marcos et al., 2019).

3. Relacionar la riqueza del sotobosque y la regeneración forestal con características significativas del rodal, responsables de la segregación del nicho de las principales especies del sotobosque (López-marcos et al., 2020).

4. Evaluar el efecto de la escala espacial sobre el área basal y la densidad del estrato arbóreo en masas mixtas vs. puras, comprender los mecanismos involucrados y analizar el efecto de la mayor área basal sobre la riqueza del sotobosque (López-Marcos et al., 2021a). 


\section{Material y métodos}

Se realizó un ensayo de tripletes ubicado en la "Sierra de la Demanda" (Burgos y Soria; $41^{\circ} 47^{\prime} 35^{\prime \prime} \mathrm{N}$ y $41^{\circ} 53^{\prime} 41^{\prime \prime} \mathrm{N}$ de latitud y $2^{\circ} 56^{\prime} 12^{\prime \prime} \mathrm{W}$ y $3^{\circ} 20^{\prime} 46^{\prime \prime} \mathrm{W}$ de longitud) que contó con 18 parcelas divididas en 6 tripletes que describían un eje este-oeste de $33 \mathrm{~km}$ y un eje norte-sur de $18 \mathrm{~km}$ (Fig. 2). El clima es Templado (Csb y Cfb) según la clasificación de Köppen (1936) para la Península Ibérica. La temperatura media anual oscila entre 8,7 y $9,8^{\circ} \mathrm{C}$ y la precipitación anual oscila entre 684 y $833 \mathrm{~mm}$. La altitud varía de 1093 a 1277 msnm y la pendiente de 0,9 a 20\%. Los materiales parentales geológicos son areniscas y margas de la era Mesozoica (IGME, 2015). Los suelos son Inceptisoles con un régimen de humedad del suelo xérico y un régimen de temperatura mésico y se clasifican como Dystroxerept Typic o Typic Humixerept (sensu Soil-Survey-Staff, 2014). La textura del suelo es predominantemente arenosa y el pH varía de extremadamente ácido a muy ácido (López-Marcos et al., 2018, 2021b). La vegetación natural que rodea la zona de estudio, altamente degradada por la acción antropogénica, se caracteriza por melojares (Quercus pyrenaica Willd.) y sabinares o enebrales (Juniperus thurifera L. y Juniperus oxycedrus L.).

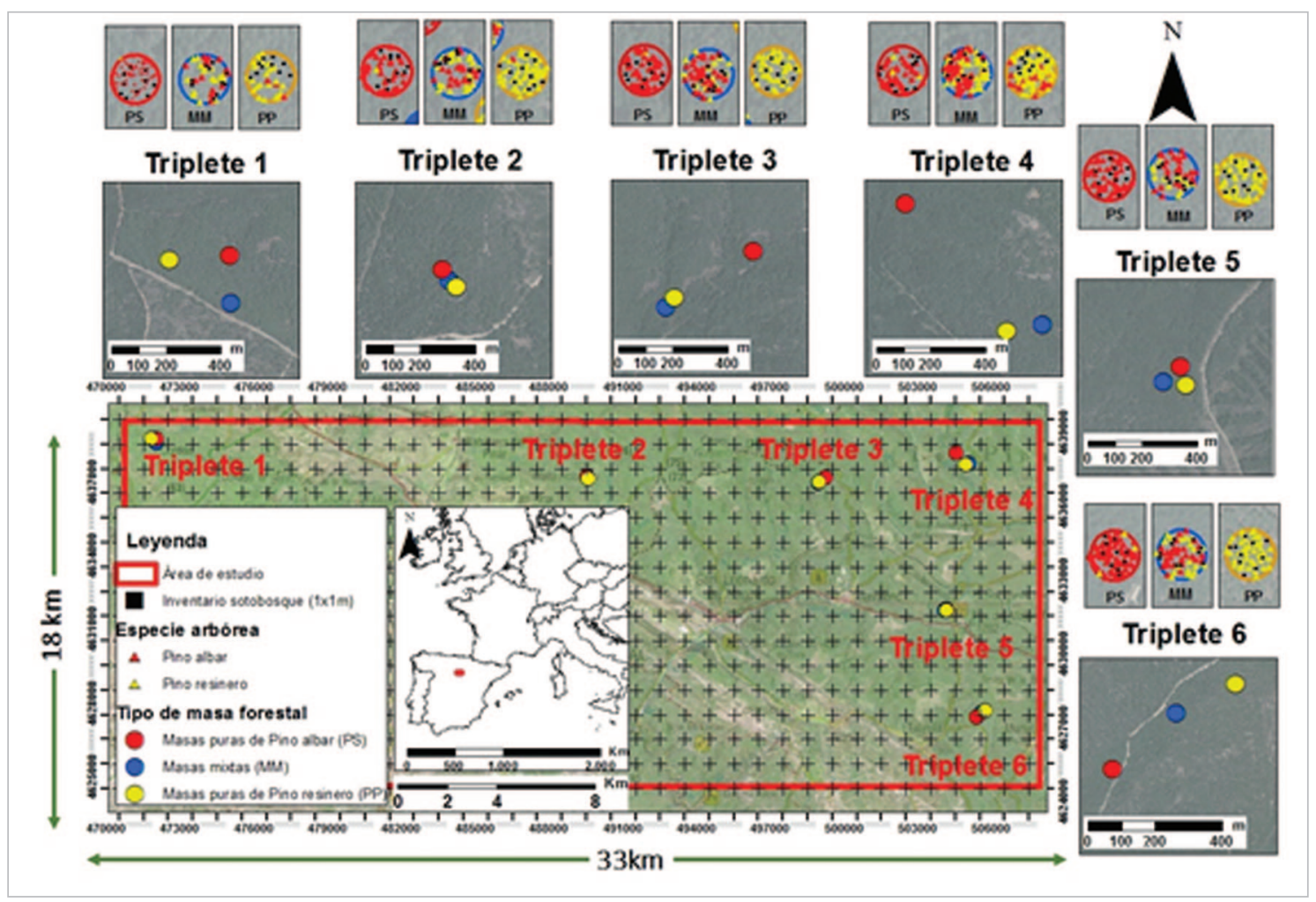

Figura 2. Localización de los tripletes en la 'Sierra de la Demanda' en la península Ibérica, parcelas dentro de cada triplete (círculos rojos: parcelas puras de pino albar, PS; círculos amarillos: parcelas puras de pino resinero, PP; círculos azules: parcelas mixtas de ambas especies de pinos, MM), los inventarios del sotobosque (pequeños cuadrados negros), y árboles (pino albar: pequeños triángulos rojos; pino resinero: pequeños triángulos amarillos) dentro de cada parcela. (modificado de López-Marcos et al., 2019, 2021). 
Cada triplete consta de dos parcelas monoespecificas de $P$. sylvestris o $P$. pinaster y una parcela mixta de ambas especies. En cada parcela, se estudió:

a. El suelo con una calicata (50 $\mathrm{cm}$ de profundidad), caracterizando los horizontes orgánicos y minerales.

b. El sotobosque con diez inventarios $(1 \mathrm{x} 1 \mathrm{~m})$ por parcela, donde se estimó la cobertura por especies (codificada después por biotipos de Raunkier) y la regeneración forestal (número individuos).

c. El estrato arbóreo a dos escalas espaciales (pequeña escala: 10 inventarios de $4 \mathrm{~m}$ de radio por parcela; nivel de rodal: un inventario de $15 \mathrm{~m}$ de radio por parcela), evaluando densidad y área basal total (López-Marcos, 2020; LópezMarcos et al., 2021b).

\section{Resultados y discusión}

Cuando se estimó el almacenamiento de carbono (Cstock) y los cationes intercambiables del perfil edáfico, se encontraron dos tendencias: en la capa superior del suelo $(0-10 \mathrm{~cm})$, Cstock fue mayor en las masas de $P$. sylvestris, menor en las de $P$. pinaster e intermedios en las mixtas; este patrón se relacionó con el ratio $\mathrm{C} / \mathrm{N}$ de la hojarasca. En las capas subsuperficiales $(10-30 \mathrm{~cm})$, el Cstock fue más alto en las masas mixtas y se relacionó con el mayor porcentaje de raíces finas y el mayor espesor del primer horizonte mineral. Las diferencias en los cationes intercambiables se relacionaron con el carbono orgánico (Fig. 3; López-Marcos et al., 2018).

Cuando se evaluó la riqueza y composición del sotobosque y su relación con el status edáfico, un gradiente de estrés hídrico, asociado a la composición del estrato arbóreo, indicó que $P$ pinaster toleraba menor contenido de agua edáfico que $P$. sylvestris. Las masas mixtas, bajo mayor estrés hídrico que las puras de $P$. sylvestris, eran capaces de mantener la riqueza del sotobosque. Además, se identificó un gradiente de fertilidad edáfica definido por el carbono orgánico y las reservas de magnesio intercambiable. Los hemicriptófitos, más abundantes en las masas mixtas, fue el único biotipo del sotobosque relacionado positivamente con la fertilidad del suelo (Fig. 3; López-Marcos et al., 2019).

Cuando se analizó la regeneración forestal y el sotobosque (composición de especies), el porcentaje de área basal de ambas especies de Pinus fue la única característica del rodal que influyó significativamente en la composición de especies del sotobosque y en la regeneración. Especies características de zonas húmedas y templadas (incluido el regenerado de $P$. sylvestris), dominaban en las masas puras de $P$. sylvestris, y especies típicas de áreas mediterráneas bien drenadas (incluido el regenerado de $P$. pinaster) dominaban en las masas puras de $P$. pinaster. En las masas mixtas, la regeneración del roble endémico, Quercus pyrenaica Willd., fue mayor y estuvo acompañada de especies del sotobosque con las que comparte nicho (Fig. 3; López-marcos et al., 2020). 


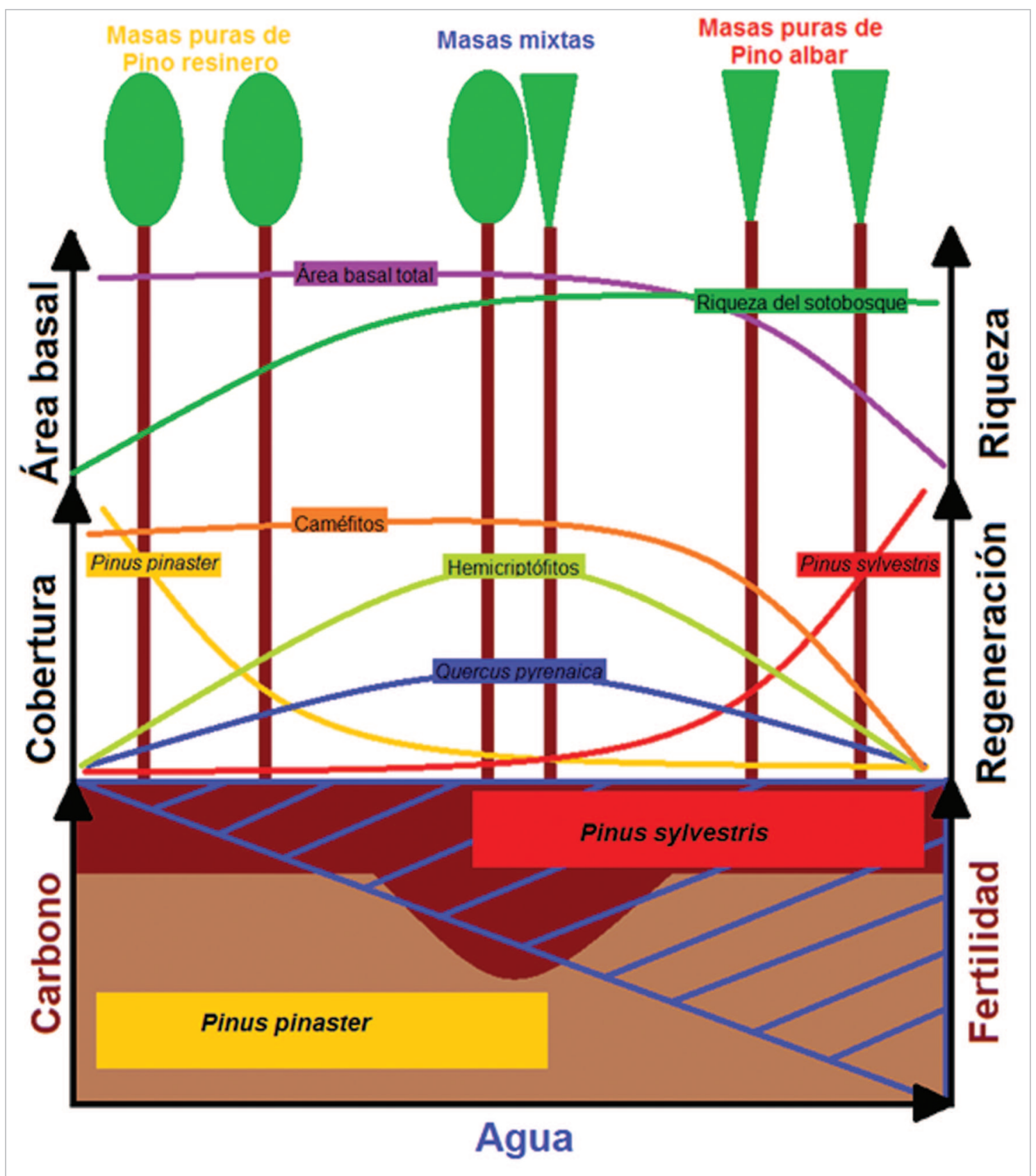

Figura 3. Resumen gráfico de la tesis doctoral. (modificado de López-Marcos 2020).

Cuando se estudió la productividad forestal (a dos escalas espaciales) se relacionó el status edáfico y se analizó su repercusión en el sotobosque, se encontró un exceso de área basal total en las masas mixtas (sólo a pequeña escala) y se relacionó con la mayor eficiencia del uso del espacio de ambos pinos, gracias a la complementariedad de nicho edáfico (agua y fertilidad). Además, el exceso de área basal total no tuvo un efecto negativo sobre la riqueza del sotobosque en las masas mixtas gracias a la contribución de los hemicriptófitos (Fig. 3; López-Marcos et al., 2021a). 


\section{Conclusiones}

Parece que la mezcla de $P$. sylvestris y $P$. pinaster supone una ventaja competitiva sobre las masas monoespecíficas en el suministro de servicios ecosistémicos, teniendo en cuenta, que estas mezclas están ampliamente distribuidas en España, parece adecuado proponer que se sigan potenciando en el área de estudio porque contribuyen a incrementar la acumulación de carbono en el subsuelo, proporcionar un exceso de área basal total, conservar la regeneración de especies endémicas y mantener la riqueza del sotobosque en suelos con menor contenido hídrico.

\section{Agradecimientos y financiación}

Me gustaría agradecer a las Dras. Carolina Martínez-Ruiz y Ma Belén Turrión Nieves por guiarme y dirigir esta tesis con un enorme cariño y profesionalidad, al Dr. Felipe Bravo por confiar en mi para desarrollar este trabajo, a Luis Alfonso Ramos Calvo por su inestimable ayuda con el muestreo de suelos, a Carmen Blanco y Juan Carlos Arranz por su asesoramiento en los análisis de laboratorio y al Dr. José Riofrío y Cristóbal Ordoñez por su asistencia en la caracterización y ubicación de las parcelas en el campo. Esta investigación ha sido financiada por el Ministerio de Economía y Competitividad del Gobierno español concedida a FB (FORMIXING. AGL2014-51964-C2-1-R) y una beca predoctoral a DLM (BES-2015072852).

\section{Bibliografía}

Andivia, E.; Rolo, V.; Jonard, M.; Formánek, P.; Ponette, Q.; 2016. Tree species identity mediates mechanisms of top soil carbon sequestration in a Norway spruce and European beech mixed forest. A. For. Sci. 73, 437-447. https://doi.org/10.1007/s13595-015-0536-z

Beddoe, R.; Costanza, R.; Farley, J.; Garza, E.; Kent, J.; Kubiszewski, I.; Martinez, L.; McCowen, T.; Murphy, K.; Myers, N.; Ogden, Z.; Stapleton, K.; Woodward, J.; 2009. Overcoming systemic roadblocks to sustainability: The evolutionary redesign of worldviews, institutions, and technologies. Proc Natl Acad Sci 106 (8), 2483-2489. https://doi.org/10.1073/pnas.0812570106

Brandtberg, P.O.; Lundkvist, H.; Bengtsson, J.; 2000. Changes in forest-floor chemistry caused by a birch admixture in Norway spruce stands. For Ecol Manage 130, 253-264. https://doi.org/10.1016/S0378-1127(99)00183-8

Bravo-Oviedo, A.; Pretzsch, H.; Ammer, C.; Andenmatten, E.; Barbati, A.; Barreiro, S.; Brang, P.; Bravo, F.; Coll, L.; Corona, P.; den Ouden, J.; Ducey, M.J.; Forrester, D.I.; Giergiczny, M.; Jacobsen, J.B.; Lesinski, J.; Löf, M.; Mason, W.L.; Matovic, B.; Metslaid, M.; Morneau, F.; Motiejunaite, J.; O'Reilly, C.; Pach, M.; Ponette, Q.; del Rio, M.; Short, I.; Skovsgaard, J.P.; Soliño, M.; Spathelf, P.; Sterba, H.; Stojanovic, D.; Strelcova, K.; Svoboda, M.; Verheyen, K.; von Lüpke, N.; Zlatanov, T.; 2014. European mixed forests: Definition and research perspectives. For Syst 23, 518-533. https://doi.org/10.5424/fs/2014233-06256 
Cattaneo, N.; Bravo-Oviedo, A.; Bravo, F.; 2018. Analysis of tree interactions in a mixed Mediterranean pine stand using competition indices. Eur J For Res 137, 109-120. https://doi.org/10.1007/s10342-017-1094-8

Cattaneo, N.; Schneider, R.; Bravo, F.; Bravo-Oviedo, A.; 2020. Inter-specific competition of tree congeners induces changes in crown architecture in Mediterranean pine mixtures. For Ecol Manage 476,118471. https://doi.org/10.1016/j.foreco.2020.118471

Davidson, EA.; Belk, E.; Boone, R.D.; 1998. Soil water content and temperature as independent or confounded factors controlling soil respiration in a temperate mixed hardwood forest. Glob Chang Biol 4, 217-227. https://doi.org/10.1046/j.1365-2486.1998.00128.x

Del Río, M.; Pretzsch, H.; Alberdi, I.; Bielak, K.; Bravo, F.; Brunner, A.; Condés, S.; Ducey, M. J.; Fonseca, T.; von Lüpke, N.; Pach, M.; Peric, S.; Perot, T.; Souidi, Z.; Spathelf, P.; Sterba, H.; Tijardovic, M.; Tomé, M.; Vallet, P.; Bravo-Oviedo, A.; (2015) Characterization of the structure, dynamics, and productivity of mixed-species stands: review and perspectives. Eur J For Res 135, 23-49. https://doi.org/10.1007/s10342-015-0927-6

Ehrlich, P.R.; Mooney, H.A.; 1983. Extinction, substitution, and ecosystem services. Bioscience 33 (4), 248-254 https://doi.org/10.2307/1309037

EUFORGEN 2009. European forest genetic Resources programme. http://www.euforgen.org/

Forrester, D.I.; Bauhus, J.; Cowie, A.L.; 2005. On the success and failure of mixed-species tree plantations: Lessons learned from a model system of Eucalyptus globulus and Acacia mearnsii. For Ecol Manage 209, 147-155 https://doi.org/10.1016/j.foreco.2005.01.012

Forrester, D.I.; Bauhus, J.; Cowie, A.L.; 2006a. Carbon allocation in a mixed-species plantation of Eucalyptus globulus and Acacia mearnsii. For Ecol Manage 233, 275-284. https://doi.org/10.1016/j.foreco.2006.05.018

Forrester, D.I.; Bauhus, J.; Cowie, A.L.; Vanclay, J.K.; 2006b. Mixed-species plantations of Eucalyptus with nitrogen-fixing trees: A review. For Ecol Manage 233, 211-230. https://doi.org/10.1016/j.foreco.2006.05.012

Forrester, D.I.; Bauhus, J.; Khanna, P.K.; 2004. Growth dynamics in a mixed-species plantation of Eucalyptus globulus and Acacia mearnsii. For Ecol Manage 193, 81-95. https://doi.org/10.1016/j.foreco.2004.01.024

Gamfeldt, L.; Snäll, T.; Bagchi, R.; Jonsson, M.; Gustafsson, L.; Kjellander, P.; Ruiz-Jaen, M.C.; Fröberg, M.; Stendahl, J.; Philipson, C.D.; Mikusiski, G.; Andersson, E.; Westerlund, B.; Andrén, H.; Moberg, F.; Moen, J.; Bengtsson, J.; 2013. Higher levels of multiple ecosystem services are found in forests with more tree species. Nat Commun 4, 1340. https://doi.org/10.1038/ncomms2328

Granda, E.; Gazol, A.; Camarero, J.J.; 2018. Functional diversity differently shapes growth resilience to drought for co-existing pine species. J Veg Sci 29, 265-275. https://doi.org/10.1111/jvs.12617

IGME (2015) Mapa Geológico de la Península Ibérica, Baleares y Canarias a escala 1M. https://info.igme.es/cartografiadigital/geologica/Geologicos1M.aspx

Jucker, T.; Bouriaud, O.; Avacaritei, D.; D_nil_, I.; Duduman, G.; Valladares, F.; Coomes, D.A.; 2014. Competition for light and water play contrasting roles in driving diversity-productivity relationships in Iberian forests. J Ecol 102, 1202-1213. https://doi.org/10.1111/13652745.12276

Köppen W;.1936. Das geographische System der Klimate. In: Köppen, W.; Geiger, R. (eds): Handbuch der Klimatologie. Velag von Gebruder Borntraeger. Berlin. pp 7-30

Ledo, A.; Cañellas, I.; Barbeito, I.; Gordo, F.J.; Calama, R.A.; Gea-Izquierdo, G.; 2014. Species 
coexistence in a mixed Mediterranean pine forest: Spatio-temporal variability in trade-offs between facilitation and competition. For Ecol Manage 322, 89-97. https://doi.org/10.1016/j.foreco.2014.02.038

López-Marcos, D.; 2020. Ecosystem services of mixed stands of Scots pine and Maritime pine: biodiversity conservation and carbon sequestration. Tesis doctoral. Universidad de Valladolid. https://uvadoc.uva.es/handle/10324/43956

López-Marcos, D.; Martínez-Ruiz, C.; Turrión, M.B.; Jonard, M.; Titeux, H.; Ponette, Q.; Bravo, F.; 2018. Soil carbon stocks and exchangeable cations in monospecific and mixed pine forests. Eur J For Res 137, 831-847. https://doi.org/10.1007/s10342-018-1143-y

López-Marcos, D.; Turrión, M.B.; Bravo, F.; Martínez-Ruiz, C.; 2019. Understory response to overstory and soil gradients in mixed versus monospecific Mediterranean pine forests. Eur J For Res 138, 939-955. https://doi.org/10.1007/s10342-019-01215-0

López-Marcos, D.; Turrión, M.B.; Bravo, F.; Martinez-Ruiz, C.; 2020. Can mixed pines forests help maintain the understory richness through the native oak. Ann For Sci 77, 15. https://doi.org/10.1007/s13595-020-0919-7

López-Marcos, D.; Turrión, M.B.; Bravo, F.; Martínez-Ruiz, C.; 2021a. The over-yielding at a small scale in mixed vs. monospecific pine forest is a result of the soil niche complementarity: understory richness implications. Eur J For Res. 140, 777-791 https://doi.org/10.1007/s10342-021-01365-0

López-Marcos, D., Turrión, M.B.; Bravo, F.; Martínez-Ruiz, C.; 2021b. Characterization of mixed and monospecific stands of Scots pine and Maritime pine. Soil profile, physiography, climate, overstory and understory data. Ann For Sci. 78, 28. https://doi.org/10.1007/s13595021-01042-7

MAGRAMA 2012. Infraestructura de datos espaciales. https://sig.mapama.gob.es/geoportal/

MEA 2005. Millennium ecosystem assessment. Ecosystems and Human Well-Being: Synthesis. Island Press, Washington, DC (USA)

Montero. G.; Serrada, R.; 2013. La situación de los bosques y el sector forestal en España. In: Sociedad Española de Ciencias Forestales. (ed) $6^{\circ}$ Congreso Forestal Español. LourizánPontevedra (España)

Piotto, D.; 2008. A meta-analysis comparing tree growth in monocultures and mixed plantations. For Ecol Manage 255, 781-786. https://doi.org/10.1016/j.foreco.2007.09.065

Pretzsch, H.; del Río, M.; Schütze, G.; Ammer, Ch.; Annighöfer, P.; Avdagic, A.; Barbeito, I.; Bielak, K.; Brazaitis, G.; Coll, L.; Drössler, L.; Fabrika, M.; Forrester, D.I.; Kurylyak, V.; Löf, M.; Lombardi, F.; Matovi, B.; Mohren, F.; Motta, R.; den Ouden, J.; Pach, M.; Ponette, Q.; Skrzyszewski, J.; Sramek, V.; Sterba, H.; Svoboda, M.; Verheyen, K.; Zlatanov, T.; BravoOviedo, A.; 2016. Mixing of Scots pine (Pinus sylvestris L.) and European beech (Fagus sylvatica L.) enhances structural heterogeneity and the effect increases with water availability. For Ecol Manage 373, 149-166. https://doi.org/10.1016/j.foreco.2016.04.043

Riofrío, J.; 2018. Mixed stands growth dynamics of Scots pine and Maritime pine_: species complementarity relationships and growth effects. Tesis Doctoral. Universidad de Valladolid. https://uvadoc.uva.es/handle/10324/33116

Riofrío, J.; del Río, M.; Maguire, D.; Bravo, F.; 2019. Species Mixing Effects on Height-Diameter and Basal Area Increment Models for Scots Pine and Maritime Pine. Forests 10(3), 249. https://doi.org/10.3390/f10030249

Riofrío, J.; del Río, M.; Pretzsch, H.; Bravo F.; 2017a. Changes in structural heterogeneity and stand productivity by mixing Scots pine and Maritime pine. For Ecol Manage 40, :219-228. 
https://doi.org/110.1016/j.foreco.2017.09.036

Riofrío, J.; del Río, M.; Bravo, F.; 2017b. Mixing effects on growth efficiency in mixed pine forests. Forestry 90:381-392. https://doi.org/110.1093/forestry/cpw056

Serrada, R.; Montero, G.; Reque, J.A.; 2008. Compendio de selvicultura aplicada en España. Instituto Nacional de Investigación y Tecnología Agraria y Alimentaria-INIA., Mdrid (Spain)

Soil-Survey-Staff 2014. Keys to Soil Taxonomy, 12 th. USDA-Natural Resources Conservation Service, Washington, DC (USA)

Trasobares, A.; Pukkala, T.; Miina, J.; 2004. Growth and yield model for uneven-aged mixtures of Pinus sylvestris L. and Pinus nigra Arn. in Catalonia, north-east Spain. Ann For Sci 61, 9-24. https://doi.org/10.1051/forest:2003080 